\title{
Multi-Item Fuzzy Inventory Model Involving Three Constraints: A Karush-Kuhn-Tucker Conditions Approach
}

\author{
R. Kasthuri, P. Vasanthi, S. Ranganayaki ${ }^{*}$ C. V. Seshaiah \\ Department of Mathematics, Sri Ramakrishna Engineering College, Coimbatore, India \\ E-mail: \{kasthuripremkumar, ${ }^{*}$ rakhul11107\}@yahoo.com, \{vasdev1066, cvseshaiah\}@gmail.com \\ Received July 7, 2011; revised July 22, 2011; accepted August 22, 2011
}

\begin{abstract}
In this paper, a multi-item inventory model with storage space, number of orders and production cost as constraints are developed in both crisp and fuzzy environment. In most of the real world situations the cost parameters, the objective functions and constraints of the decision makers are imprecise in nature. This model is solved with shortages and the unit cost dependent demand is assumed. Hence the cost parameters are imposed here in fuzzy environment. This model has been solved by Kuhn-Tucker conditions method. The results for the model without shortages are obtained as a particular case. The model is illustrated with numerical example.
\end{abstract}

Keywords: Multi-Item Inventory Model, Membership Function, Karush-Kuhn-Tucker Condition

\section{Introduction}

The literal meaning of inventory is the stock of goods for future use (production/sales). The control of inventories of physical goods is a problem common to all enterprises in any sector of an economy. The basic objective of inventory control is to reduce investment in inventories and ensuring that production process does not suffer at the same time.

In general the classical inventory problems are designed by considering that the demand rate of an item is constant and deterministic and that the unit price of an item is considered to be constant and independent in nature. But in practical situation, unit price and demand rate of an item may be related to each other. When the demand of an item is high, an item is produced in large numbers and fixed costs of production are spread over a large number of items. Hence the unit cost of the item decreases. .i.e., the unit price of an item inversely relates to the demand of that item. So demand rate of an item may be considered as a decision variable.

Zadeh [1] first gave the concept of fuzzy set theory. Later on, Bellman and Zadeh [2] used the fuzzy set theory to the decision-making problem. Zimmerman [3] gave the concept to solve multi objective linear programming problem. Fuzzy set theory has made an entry into the inventory control systems. Sommer [4] applied the fuzzy concept to an inventory and production sched- uling problem. Park [5] examined the EOQ formula in the fuzzy set theoretic perspective associating the fuzziness with the cost data. Hence we may impose warehouse space, cost parameters, number of orders, production cost etc, in fuzzy environment.

The Kuhn-Tucker conditions [6] are necessary conditions for identifying stationary points of a non linear constrained problem subject to inequality constraints. The development of this method is based on the Lagrangean method.

These conditions are also sufficient if the objective function and the solution space satisfy the conditions in the following Table 1.

The conditions for establishing the sufficiency of the Kuhn-Tucker conditions [7] are summarized in the following Table 2.

Kuhn-Tucker conditions, also known as Karush-KuhnTucker (KKT) conditions was first developed by W. Karush in 1939 as part of his M.S. thesis at the University of Chicago. The same conditions were developed independently in 1951 by W. Kuhn and A. Tucker.

In this paper, a multi-item, multi-objective inventory problem with shortages along with three constraints such as limited storage space, number of orders and production cost has been formulated. The unit cost is considered here in fuzzy environment. The problem has been solved by KKT conditions method. This model is illustrated by numerical example [8-11]. 
Table 1. The objective function and the solution space.

\begin{tabular}{ccc}
\hline \multirow{2}{*}{ Sense of optimization } & \multicolumn{2}{c}{ Required conditions } \\
\cline { 2 - 3 } & Objective function & Solution space \\
\hline Maximization & Concave & Convex Set \\
Minimization & Convex & Convex Set \\
\hline
\end{tabular}

Table 2. The sufficiency of the Kuhn-Tucker conditions.

\begin{tabular}{ll}
\hline \multicolumn{1}{c}{ Problem } & \multicolumn{1}{c}{ Kuhn-Tucker conditions } \\
\hline 1) Max $z=f(X)$ & $\frac{\partial}{\partial x_{j}} f(X)-\sum_{i=1}^{m} \lambda_{i} \frac{\partial}{\partial x_{j}} h^{i}(X)=0$ \\
subject to $h^{i}(X) \leq 0$ & $\lambda_{i} h^{i}(X)=0, h^{i}(X) \leq 0, i=1,2, \cdots, m$ \\
$X \geq 0, i=1,2, \cdots, m$ & $\lambda_{i} \geq 0, i=1,2, \cdots, m$ \\
& $\frac{\partial}{\partial x_{j}} f(X)-\sum_{i=1}^{m} \lambda_{i} \frac{\partial}{\partial x_{j}} h^{i}(X)=0$ \\
2) Min $z=f(X)$ & $\lambda_{i} h^{i}(X)=0, h^{i}(X) \geq 0, i=1,2, \cdots, m$ \\
subject to $h^{i}(X) \geq 0$ & $\lambda_{i} \geq 0, i=1,2, \cdots, m$ \\
$X \geq 0, i=1,2, \cdots, m$ & \\
\hline
\end{tabular}

\section{Assumptions and Notations}

A multi-item, multi-objective inventory model is developed under the following notations and assumptions.

\subsection{Notations}

$n=$ number of items

$t=$ number of orders

$W=$ Floor (or) shelf-space available

$B=$ Total investment cost for replenishment

For $i^{\text {th }}$ item: $(i=1,2, \cdots, n)$

$D_{i}=D_{i}\left(p_{i}\right)$ demand rate [function of unit cost price]

$Q_{i}=$ lot size (decision variable)

$M_{i}=$ Shortage level (decision variable)

$S_{i}=$ Set-up cost per cycle

$H_{i}=$ Inventory holding cost per unit item

$m_{i}=$ Shortage cost per unit item

$p_{i}=$ price per unit item (decision variable)

$w_{i}=$ storage space per item

$T C(p, Q, M)=$ expected annual total cost

\subsection{Assumptions}

1) replenishment is instantaneous

2) lead time is zero

3) demand is related to the unit price as

$$
D_{i}=\frac{A_{i}}{p_{i}^{\beta_{i}}}=A_{i} p_{i}^{-\beta_{i}}
$$

where $A_{i}(>0)$ and $\beta_{i}\left(0<\beta_{i}<1\right)$ are constants and real numbers selected to provide the best fit of the estimated price function. $A_{i}>0$ is an obvious condition since both $D_{i}$ and $p_{i}$ must be non-negative.

\section{Formulation of Inventory Model with Shortages}

Let the amount of stock for the $i^{\text {th }}$ item $(i=1,2, \cdots, n)$ be $R_{i}$ at time $t=0$. In the interval $\left(0, T_{i}\left(=t_{1 i}+t_{2 i}\right)\right)$, the inventory level gradually decreases to meet demands. By this process the inventory level reaches zero level at time $t_{1 i}$ and then shortages are allowed to occur in the interval $\left(t_{1 i}, T_{i}\right)$. The cycle then repeats itself (Figure 1).

The differential equation for the instantaneous inventory $q_{i}(t)$ at time $\mathrm{t}$ in $\left(0, T_{i}\right)$ is given by

$$
\begin{aligned}
\frac{d q_{i}(t)}{d t} & =-D_{i} \text { for } 0 \leq t \leq t_{1 i} \\
& =-D_{i} \text { for } t_{1 i} \leq t \leq T_{i}
\end{aligned}
$$

with the initial conditions $q_{i}(0)=R_{i}\left(=Q_{i}-M_{i}\right), q_{i}\left(T_{i}\right)=$ $-M_{i}, q_{i}\left(t_{1 i}\right)=0$.

For each period a fixed amount of shortage is allowed and there is a penalty cost $m_{i}$ per items of unsatisfied demand per unit time.

From (1)

$$
\begin{aligned}
& q_{i}(t)=R_{i}-D_{i} t \text { for } 0 \leq t \leq t_{1 i} \\
& =D_{i}\left(t_{1 i}-t\right) \text {. for } t_{1 i} \leq t \leq T_{i} \\
& \text { So } D_{i} t_{1 i}=R_{i}, M_{i}=D_{i} t_{2 i}, Q_{i}=D_{i} T_{i} \\
& \text { Holding cost }=H_{i} \int_{0}^{t_{1 i}} q_{i}(t) \mathrm{d} t=\frac{H_{i}\left(Q_{i}-M_{i}\right)^{2}}{2 Q_{i}} T_{i} \\
& \text { Shortage cost }=-m_{i} \int_{t_{1 i}}^{T_{i}} q_{i}(t) \mathrm{d} t=\frac{m_{i} M_{i}^{2}}{2 Q_{i}} T_{i} \\
& \text { Production cost }=p_{i} Q_{i} \\
& \text { The total cost }=\text { Production cost }+ \text { Set up cost } \\
& + \text { Holding cost }+ \text { Shortage cost } \\
& =p_{i} Q_{i}+S_{i}+H_{i} \frac{\left(Q_{i}-M_{i}\right)^{2}}{2 Q_{i}} T_{i}+\frac{m_{i} M_{i}^{2}}{2 Q_{i}} T_{i}
\end{aligned}
$$

The total average cost of the $i^{\text {th }}$ item is

$$
\begin{gathered}
T C\left(p_{i}, Q_{i}, M_{i}\right)=p_{i} D_{i}+\frac{S_{i} D_{i}}{Q_{i}}+\frac{H_{i}\left(Q_{i}-M_{i}\right)^{2}}{2 Q_{i}}+\frac{m_{i} M_{i}^{2}}{2 Q_{i}} \\
T C_{i}\left(p_{i}, Q_{i}, M_{i}\right)= \\
A_{i} p_{i}^{1-\beta_{i}}+\frac{A_{i} S_{i}}{Q_{i}} p_{i}^{-\beta_{i}} \\
+\frac{H_{i}\left(Q_{i}-M_{i}\right)^{2}}{2 Q_{i}}+\frac{m_{i} M_{i}^{2}}{2 Q_{i}}
\end{gathered}
$$

for $i=1,2,3, \cdots, n$. 


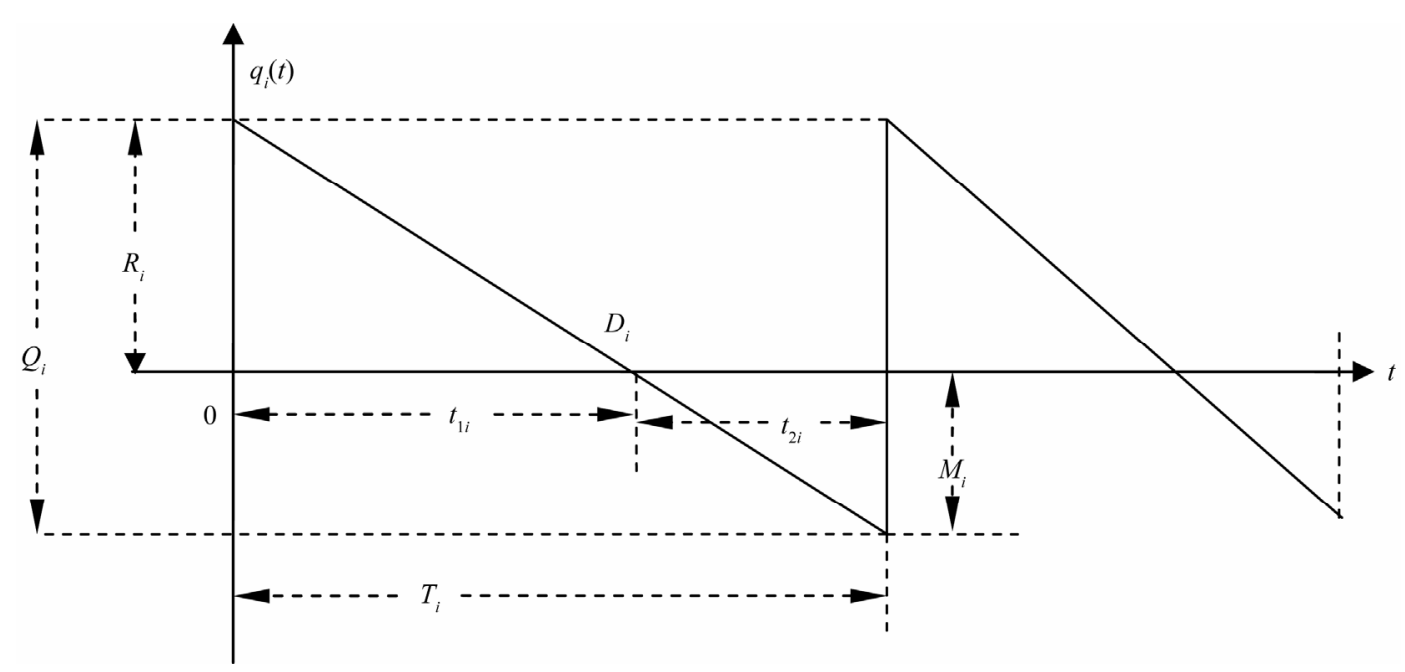

Figure 1. Inventory level of the $i^{\text {th }}$ item.

There are some restrictions on available resources in inventory problems that cannot be ignored to derive the optimal total cost.

1) There is a limitation on the available warehouse floor space where the items are to be stored i.e.

$\sum_{i=1}^{n} w_{i} Q_{i} \leq W$

2) Investment amount on total production cost cannot be infinite, it may have an upper limit on the maximum investment. i.e. $\sum_{i=1}^{n} p_{i} Q_{i} \leq B$;

3) An upper limit on the number of orders that can be made in a time cycle on the system (i.e.) $\sum_{i=1}^{n} \frac{D_{i}}{Q_{i}} \leq t$.

The problem is to find price per unit item, the lot size, the shortage amount so as to minimize the total average cost function (2) subject to the total space and total production cost restrictions.

It may be written as

Min $T C_{i}\left(p_{i}, Q_{i}, M_{i}\right)$ for all $i=1,2,3, \cdots, n$.

Subject to the inequality constraints

$$
\begin{gathered}
\sum_{i=1}^{n} w_{i} Q_{i} \leq W \\
\sum_{i=1}^{n} p_{i} Q_{i} \leq B \\
\sum_{i=1}^{n} \frac{D_{i}}{Q_{i}} \leq t
\end{gathered}
$$

\section{Fuzzy Inventory Model with Shortages}

When $p_{i^{\prime}}^{s}$ are fuzzy decision variables, the above crisp model under fuzzy environment reduces to

$$
\begin{aligned}
\operatorname{MinTC}(\tilde{p}, Q, M)= & \sum_{i=1}^{n}\left[A_{i} \tilde{p}_{i}^{1-\beta_{i}}+\frac{A_{i} S_{i}}{Q_{i}} \tilde{p}_{i}^{-\beta_{i}}\right. \\
& \left.+\frac{H_{i}\left(Q_{i}-M_{i}\right)^{2}}{2 Q_{i}}+\frac{m_{i} M_{i}^{2}}{2 Q_{i}}\right] \leq t
\end{aligned}
$$

subject to the constraints

$$
\begin{gathered}
\sum_{i=1}^{n} w_{i} Q_{i} \leq W \\
\sum_{i=1}^{n} \tilde{p}_{i} Q_{i} \leq B \\
\sum_{i=1}^{n} \tilde{p}_{i}^{1-\beta_{i}} A_{i} Q_{i}^{-1} \leq t
\end{gathered}
$$

[Here cap ' $\sim$ ' denotes the fuzzification of the parameters.]

The above fuzzy non-linear programming can be solved using Kuhn-Tucker conditions.

\subsection{Membership Function}

The membership function for the fuzzy variable $p_{i}$ is defined as follows

$$
\mu_{p_{i}}(X)=\left\{\begin{array}{l}
1, p_{i} \leq L_{L_{i}} \\
\frac{U_{L_{i}}-p_{i}}{U_{L_{i}}-L_{L_{i}}}, L_{L_{i}} \leq p_{i} \leq U_{L_{i}} \\
0, p_{i} \geq U_{L_{i}}
\end{array}\right\}
$$

Here $U_{L i}$ and $L_{L i}$ are upper limit and lower limit of $p_{i}$ respectively. 


\subsection{Fuzzy Inventory Model without Shortages}

When $p_{i^{\prime}}^{s}$ are fuzzy decision variables, the above crisp model without shortages under fuzzy environment reduces to

$$
\operatorname{Min} T C(\tilde{p}, Q)=\sum_{i=1}^{n}\left[A_{i} \tilde{p}_{i}^{1-\beta_{i}}+\frac{A_{i} S_{i}}{Q_{i}} \tilde{p}_{i}^{-\beta_{i}}+\frac{H_{i} Q_{i}}{2}\right]
$$

subject to the constraints

$$
\begin{gathered}
\sum_{i=1}^{n} w_{i} Q_{i} \leq W \\
\sum_{i=1}^{n} \tilde{p}_{i} Q_{i} \leq B \\
\sum_{i=1}^{n} \tilde{p}_{i}^{-\beta_{i}} A_{i} Q_{i}^{-1} \leq t
\end{gathered}
$$

\section{Numerical Example}

To solve the above non-linear programming using KuhnTucker conditions, the following values are assumed.

$$
\begin{gathered}
n=1, t=3 A_{1}=100, S_{1}=\$ 100, H_{1}=\$ 1, \\
w_{1}=2 \text { sq. } \mathrm{ft}, W=150 \text { sq. } \mathrm{ft}, B=\$ 1200, \\
m_{1}=\$ 1 \text { and } \$ 10 \leq p_{1} \leq \$ 20
\end{gathered}
$$

By the method of Kuhn-Tucker conditions, consider the four cases

1) $\lambda_{1}=0, \lambda_{2}=0$

2) $\lambda_{1} \neq 0, \lambda_{2}=0$

3) $\lambda_{1}=0, \lambda_{2} \neq 0$

4) $\lambda_{1} \neq 0, \lambda_{2} \neq 0$

Here Kuhn-Tucker conditions are used as trial and error method by taking different values for $\beta_{1}$ until an optimum result is obtained.

Optimal solutions for the fuzzy model with shortages

\begin{tabular}{ccccccc}
\hline$\beta_{1}$ & $p_{1}$ & $\begin{array}{c}\mu_{P 1} \\
\text { value }\end{array}$ & $Q_{1}$ & $D_{1}$ & $M_{1}$ & $\begin{array}{c}\text { Expected } \\
\text { Total cost }\end{array}$ \\
\hline 0.88 & 10.179 & 0.982 & 72.046 & 12.978 & 36.023 & 168.133 \\
0.89 & 12.406 & 0.759 & 65.220 & 10.85 & 32.609 & 164.484 \\
0.90 & 15.405 & 0.4595 & 58.428 & 8.533 & 29.214 & 160.664 \\
0.91 & 19.561 & 0.0439 & 51.693 & 6.681 & 25.847 & 156.533 \\
\hline
\end{tabular}

From the above table it follows that 10.179 has the maximum membership value 0.982 .

Hence the required optimum solution is

$$
p_{1}=10.179, Q_{1}=72.046, M_{1}=36.023
$$

Minimum expected Total cost $=\$ 168.133$.

Optimal solutions for the fuzzy model without shortages

\begin{tabular}{cccccc}
\hline$\beta_{1}$ & $p_{1}$ & $\begin{array}{c}\mu_{P 1} \\
\text { value }\end{array}$ & $Q_{1}$ & $D_{1}$ & $\begin{array}{c}\text { Expected } \\
\text { Total cost }\end{array}$ \\
\hline 0.89 & 10.788 & 0.921 & 75.000 & 12.042 & 183.460 \\
0.85 & 11.178 & 0.882 & 50.693 & 12.850 & 194.327 \\
0.92 & 16.00 & 0.400 & 75.000 & 07.802 & 172.733 \\
\hline
\end{tabular}

From the above table it follows that 10.788 has the maximum membership value 0.921 .

Hence the required optimum solution is

$$
p_{1}=10.788, Q_{1}=75
$$

Minimum expected Total cost $=\$ 183.460$.

\section{Conclusions}

In this paper we have proposed a concept of the optimal solution of the inventory problem with fuzzy cost price per unit item. Fuzzy set theoretic approach of solving an inventory control problem is realistic as there is nothing like fully rigid in the world. By solving the above fuzzy inventory model using Kuhn-Tucker condition method we have the values of imprecise variable for decision making. The above discussed model can be developed with many limitations, such as their inventory level, Warehouse space and budget limitations, etc.

\section{References}

[1] L. A. Zadeh, "Fuzzy Sets," Information and Control, Vol. 8, No. 3, 1965, pp. 338-353. doi:10.1016/S0019-9958(65)90241-X

[2] R. E. Bellman and L. A. Zadeh, "Decision-Making in a Fuzzy Environment," Management Science, Vol. 17, No. 4, 1970, pp. B141-B164. doi:10.1287/mnsc.17.4.B141

[3] H. J. Zimmermann, "Description and Optimization of Fuzzy Systems," International Journal of General Systems, Vol. 2, No. 4, 1976, pp. 209-215. doi: $10.1080 / 03081077608547470$

[4] G. Sommer, "Fuzzy Inventory Scheduling," In: G. Lasker, Ed., Applied Systems and Cybernetics, Vol. 6, Academic Press, New York, 1981.

[5] K. S. Park, "Fuzzy Set Theoretic Interpretation of Economic Order Quantity," IEEE Transactions on Systems, Man, and Cybernetics, Vol. 17, No. 6, 1987, pp. 1082-1084.

[6] H. A. Taha, "Operations Research: An Introduction," Prentice-Hall of India, Delhi, 2005, pp. 725-728.

[7] P. K. Gupta and M. Mohan, "Problems in Operations Research (Methods \& Solutions)," Sultan Chand Co., New Delhi, 2003, pp. 609-610.

[8] E. A. Silver and R. Peterson, "Decision Systems for Inventory Management and Production Planning," John Wiley, New York, 1985.

[9] H. Tanaka, T. Okuda and K. Asai, "On Fuzzy Mathe- 
matical Programming," Journal of Cybernetics, Vol. 3, No. 4, 1974, pp. 37-46. doi:10.1080/01969727308545912

[10] F. E. Raymond, "Quantity and Economic in Manufac- turer," McGraw Hill Book Co., New York, 1931.

[11] G. Hadley and T. M. Whitin, "Analysis of Inventory Systems," Prentice-Hall, Englewood Cliffs, 1958. 